\title{
Retinal Area Segmentation using Adaptive Superpixalation and its Classification using RBFN
}

\author{
Nimisha, Rana Gill \\ Department of Electronics and Comunication Engineering, Chandigarh University, Gharuan, India
}

\begin{tabular}{l}
\hline \hline Article Info \\
\hline Article history: \\
Received May 19, 2016 \\
Revised Jul 28, 2016 \\
Accepted Aug 11, 2016 \\
\hline
\end{tabular}

Keyword:

Extraction of retinal area Feature generation Machine learning approach Superpixel (SLICO)

\begin{abstract}
Retinal disease is the very important issue in medical field. To diagnose the disease, it needs to detect the true retinal area. Artefacts like eyelids and eyelashes are come along with retinal part so removal of artefacts is the big task for better diagnosis of disease into the retinal part. In this paper, we have proposed the segmentation and use machine learning approaches to detect the true retinal part. Preprocessing is done on the original image using Gamma Normalization which helps to enhance the image that can gives detail information about the image. Then the segmentation is performed on the Gamma Normalized image by Superpixel method. Superpixel is the group of pixel into different regions which is based on compactness and regional size. Superpixel is used to reduce the complexity of image processing task and provide suitable primitive image pattern. Then feature generation must be done and machine learning approach helps to extract true retinal area. The experimental evaluation gives the better result with accuracy of $96 \%$.
\end{abstract}

Copyright () 2016 Institute of Advanced Engineering and Science. All rights reserved.

\section{Corresponding Author:}

Nimisha,

Department of Electronics and Communication Engineering,

Chandigarh University,

National Highway 95, Chandigarh-Ludhiana Highway, Sahibzada Ajit Singh Nagar, Punjab 140413, India.

Email: nimisha25.singh@gmail.com

\section{INTRODUCTION}

Nowadays, Biometric systems are becoming suitable for conventional methods such as PIN, password etc. Biometric techniques are depending upon behavioural or physiological trait like hand geometry, blood vessel patterns, gait, signature and iris etc. Iris patterns consist of unique information such as ridges, crypts, corona, furrows, rings, a zigzag collarette and freckles etc. The accuracy of Iris recognition depends upon its segmentation. Iris segmentation is affected by the region of eyelashes and eyelids. The eyelashes hide eyelid boundaries so eyelid detection is difficult. But eyelashes can be detected and eliminated by the wavelet transform [1].

The diseases like age-related muscular degeneration (AMD) and diabetic retinopathy (DR) affect a large number of population and also it is expected to these diseases must be increased in our coming future. Generally, Digital fundus photography is used to screen and identify the nature of retina related condition which is possible to allow image storage, in a non invasive examination and for the transmission at different location. So, the digital retinal images are examined by an expert human grader i.e, Optometrists and Opthalmogists which perform the whole process that is time consuming and difficult [2]. Automated analysis of retinal images have the ability to reduce the time and also it must be detected the problem of the retinal part very easily. Retinal area obtained from the imaging instruments such as fundus camera and scanning laser opthalmoscope (SLO) which contain structure of retinal area with artefacts (eyelashes and eyelids) [3].

Removal of artefact is the important step before the detection of retinal diseases. Extraneous object like eylashes, dust and eyelids on the optical surfaces may come in focus and also appear bright. Extraction 
of true retinal area from the SLO images is important for the diagnosis of eye diseases. This is very difficult to differentiate between the artefacts and the true retinal area. The SLO images of retina that is obtained from optos [4] with the width of $200^{\circ}$ approximately. Figure 1 shows that the retinal images which is captured using SLO and fundus camera. With the retinal area eyelash and eyelid structures are also imaged due to the large field of view (FOV) in the SLO images. If eyelids and eyelashes are removed then analysis of retinal area along with the diagnosis of diseases in the visible retina can be done. In this paper, we have done the segmentation part and classifier construction which is help to extract the retinal area.Then diagnosis of retinal part can be easily done.

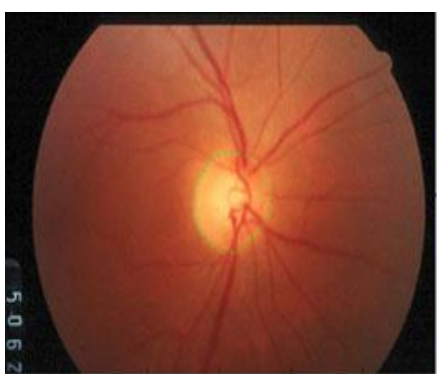

(a)

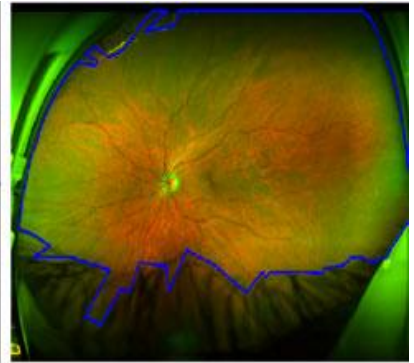

(b)

Figure 1. (a) A Fundus Image, (b) An SLO Image with True Retinal Area [3]

The main four steps are done in this paper:

a. Preprocessing task of SLO images. Preprocessing is done with Gamma Normalization.

b. Generation of Superpixel of the Gamma Normalized image. Superpixel will be generated by simple linear iterative clustering (SLIC).

c. After generating the superpixel, feature generation (textural feature and gradient feature) is done.

d. For classification of true retinal area from SLO images, construction of classifier is important.

The paper is arranged as follows. Section II shows our proposed methods which contain preprocessing, Superpixel generation, feature generation and construction of classifier. Section III provides the experimental and visual result of our proposed method. And at last section IV shows the discussion about result and the conclusion.

\section{PROPOSED METHOD}

\subsection{Image Acquisition}

Image acquisition is the very basic and important step. It gives the acquisition of image data with the annotation around the actual detection part of the images. Any method or process can be applied on the images. Images can be collected from various field like biomedical, satellite, plant etc. These images can be obtained using any digital equipment like camera, laptop, mobile phones etc. Six standard images have been taken from the dataset [9]. For the finding of results, image acquisition must be required in the field of image processing [5].

\subsection{Gamma Normalization}

Gamma Normalization is a non-linear operation which is used to control the total brightness of the given image. It is also known as Gamma correction or Power law transformation and is defined as

$$
I_{\text {input }}=I_{\text {output }}^{\mathrm{X}}
$$

where $\gamma$ is gamma. Generally, Gamma value range is in between 0 and 1 i.e, $0 \leq \gamma \leq 1$, when $\gamma \leq 1$ then the output image is brighter and it is called as an encoding gamma whereas if $\mathrm{\gamma} \geq 1$ then the output image is darker and it is called as decoding gamma and if $\gamma=1$ then it is linear. [6] Various step of enhancement can be generated for the different values of $\gamma$. Gamma Normalization can brighten the intensities of any image. It can also be used for enhance the contrast of images which has low intensity value. If $\gamma<1$, then the gamma correction forms the small range of dark pixel value to the large range and the larger range of bright pixel value into the small range. It can be written as 
$\mathrm{S}=\mathrm{cr} r^{\gamma}$

where $\mathrm{c}$ and $\mathrm{y}$ are positive constant value.

From the above equation, s versus $r$ can be plotted for different value of $\gamma$ which is shown in the Figure 2. The above Figure 2 shows that the values $\gamma>1$ have the opposite effect as the values $\gamma<1$. When $\mathrm{c}=\mathrm{\gamma}=1$, it reduces the identity transformation. So, the gamma correction is used to rectify the power law response phenomena [7]. In this paper, Gamma adjustment is used to bring the mean intensity of the image to the target value. So, Gamma can be calculated by

$$
\mathrm{\gamma}=\log _{10}\left(\mu_{\text {target }}\right)-\log _{10}(255) / \log _{10}\left(\mu_{\text {orig }}\right)-\log _{10}(255)
$$

where, $\mu_{\text {orig }}=$ mean intensity of original image, $\mu_{\text {target }}=$ mean intensity of target image and $\mu_{\text {target }}=$ mean intensity of target image. In this paper, $\mu_{\text {target }}$ is set to 80 for the image visualization and thus the gamma adjustment is

$$
I_{\text {norm }}=\left(\frac{I}{255}\right)^{\mathrm{X}}
$$

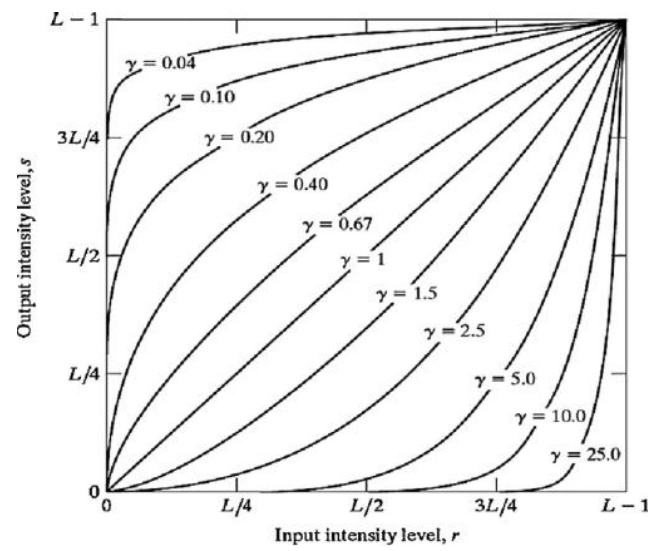

Figure 2. Plot of Equation (2) for Various Value of $\mathrm{Y}(\mathrm{c}=1$ for all) [7]

\subsection{Superpixel Generation}

After the gamma normalization, the next step is to generate the superpixel. The algorithm which is used to group all the pixels into the different region is called superpixel algorithm which is used to calculate the feature of the image and it is also used to reduce the complexity of the image processing task. Superpixel are used to capture image redundancy and support the convenient primitive image pattern. [3] In this paper, Superpixel is generated by the adaptive SLIC (SLICO) which is similar to the SLIC. SLIC uses some compactness parameter which is chosen by user for every superpixel in the image. If image is smooth at particular region but in the other region, it is highly textured, then the SLIC generate smooth regular-sized superpixels at the smooth region and at the highly textured region, SLIC produces highly irregular superpixels. So, it becomes more complicated to select the right parameter for each image. But in the SLICO, the user need not to select to select the compactness parameter. SLICO adaptively select the compactness parameter for each and every superpixel differently. For both textured and non-textured regions, SLICO used to generate regular shaped superpixels, And also SLICO is very fast as compared to SLIC.In the given Figure 3, the images in the top row shows a constant compactness factor for every superpixels but in the bottom row images show SLICO output which is adaptively chosen the compactness factor for every superpixel [8].

The two main points are there for adaptation of superpixel generation:

a. By limiting the search space at the region which is proportional to the superpixel size is reduced by the number of distance calculation.

b. By combining the spatial and colour proximity, the weighted distance can be measured. And that weighted distance use to control over the compactness and the size of Superpixel [9]. 

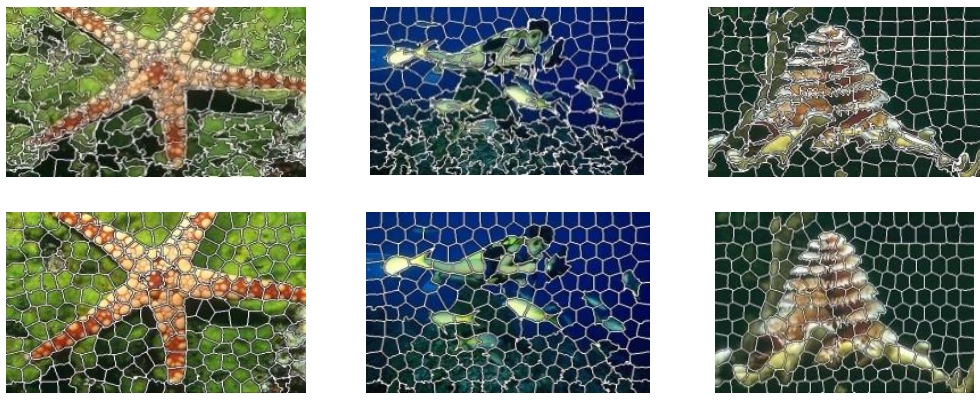

Figure 3. Upper figure shows SLIC and lower fig shows SLICO [8]

\subsubsection{Algorithm}

(1) SLIC is simple algorithm to use. In this algorithm only one parameter is used i.e, $\mathrm{k}$ which has equal sized Superpixels. The clustering procedure starts with an initialization step for the colour images in CIELAB colour space where k starting cluster centre $C_{i}=\left[l_{i} a_{i} b_{i} x_{i} y_{i}\right]$ that is sampled on the common grid spaced $S$ pixels. The grid interval $S=\sqrt{ } \mathrm{N} / \mathrm{k}$ is to produce roughly same sized superpixels. The centres are changed their position to the smallest gradient position in the $3 \times 3$ neighbourhood. The next step is the assignment step. In this step each pixel $\mathrm{i}$ is connected with nearest cluster centre in which location is overlap with search region. The size of the search region is limiting which decreases the number of distance calculation and hence the result in speed is higher as compared to traditional k-mean clustering in which all the cluster centre compared with each pixel. So the spatial extent of the region whose size is $\mathrm{S} \times \mathrm{S}$, similar pixel can be searched in the region of $2 \mathrm{~S} \times 2 \mathrm{~S}$ over the superpixel centre. When each pixel is connected with the nearest cluster centre then the clusters centres to the mean [1 a b x y] vector can be adjusted by an update step for all the pixels which belongs to the cluster. A residual error $\mathrm{E}$ is computed by the $L_{2}$ norm between the prior cluster centre locations and the recent cluster centre locations. The update and the assignment steps may be repeated iteratively till the error reduces. At last, a post processing step is done by using the reassigning disjoint pixels towards the nearby superpixels [9] as shown in Figure 1.
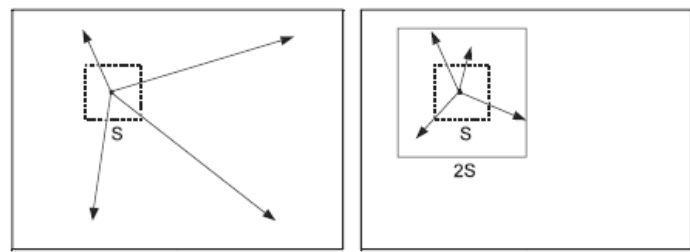

Figure 4. Standard K-Mean Searches and SLIC Searches a Limited the Entire Image Regions [9]

\subsubsection{Distance Measure}

SLIC Superpixel used to form a group in the plane space of labxy colour image. This is the problem for determining the distance measure $\mathrm{D}$. D calculates the distance between the cluster centre $C_{k}$ and a pixel $\mathrm{i}$. In the CIELAB colour space $[l a b]^{T}$, the colour of the pixels represent whose range value is known. On the other side, the pixel position $\left[\begin{array}{ll}x & y\end{array}\right]^{T}$ can use the value range vary according to the image size [9].

Simply D is defined as the 5D Euclidean distance in the space of labxy that will cause inconsistencies in clustering superpixel sizes. Spatial distances dominate colour proximity for large superpixel to give more relative value to spatial proximity than colour. The converse is true for small superpixel. It is essential to normalize spatial proximity and colour proximity by their specific maximum distances within the cluster, $N_{s}$ and $N_{c}$ and then combine the two distances, after this D will be:

$$
\begin{aligned}
& d_{c}=\sqrt{ }\left(l_{j}-l_{i}\right)^{2}+\left(a_{j}-a_{i}\right)^{2}+\left(b_{j}-b_{i}\right)^{2} \\
& d_{s}=\sqrt{ }\left(x_{j}-x_{i}\right)^{2}+\left(y_{j}-y_{i}\right)^{2} \\
& \mathrm{D}^{\prime}=\sqrt{ }\left(d_{c / N_{c}}\right)^{2}+\left(d_{s / N_{s}}\right)^{2}
\end{aligned}
$$


The maximum colour distance $N_{c}$ determination is not straight forward. This problem can be eliminated by fixing $N_{c}$ to the constant m. So D' becomes:

$$
\mathrm{D}^{\prime}=\sqrt{ }\left(d_{c / m}\right)^{2}+\left(d_{s / s}\right)^{2}
$$

\subsection{Feature Genaeration}

The next step is feature generation to determine the feature after the the generation of superpixel. The artefacts and the reinal area can diffentiated by textural and gradient based features. By green and the red channels on different smoothing scales, Textural and gradient features are calculated [10]. Blue channel must be zero in SLO images so there is no feature generated for the blue region. Textural feature and the gradient feature are described below:

a. Textural features: The textures are examined by statistical method that is gray level co-occurrence matrix (GLCM). GLCM function determine how often the intensity value $i$ take place with the adjacent intensity value $\mathrm{j}$. The adjacent pixel are observed by vthe four angles i.e, $\theta=0^{\circ}, 45^{\circ}, 90^{\circ}, 135^{\circ}$. This is shown in Figure 5. Offset value is the pixel adjacency by the particular distance. In this paper the offset value is kept as 1. GLCM calculate variousfeatures but for reductiuon of computational complexity this paper extracted only four features from GLCM i.e, contrast, correlation, energy and homogenity which is shown in the Table1.

b. Gradient features- Gradient features are necessary to calculate because of the non-uniformity of the artefacts. The response of gradient feature calculated from the gaussian filter bank [10]. It contains Gaussian $\mathrm{N}(\boldsymbol{\sigma})$, first order derivatives $N_{x}(\boldsymbol{\sigma})$ and $N_{y}(\boldsymbol{\sigma})$ and second order derivative $N_{x x}(\boldsymbol{\sigma}), N_{x y}(\boldsymbol{\sigma})$ and $N_{y y}(\boldsymbol{\sigma})$, The mean value is calculated by the gaussian filter bank over each and every superpixel in all the pixels. Mean standard deviation and varience are calculated in this paper which is shown in Table 2.

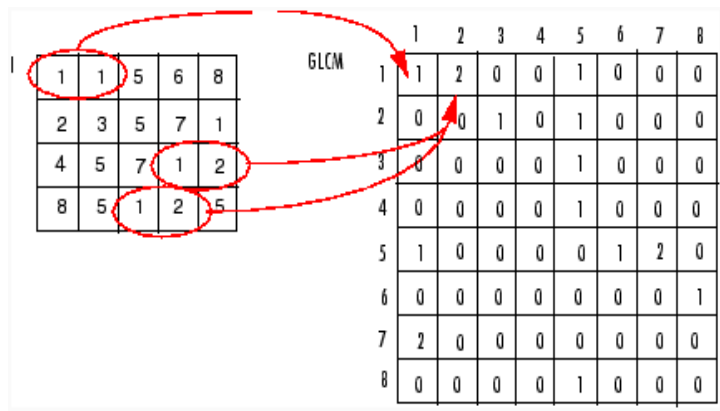

(a)

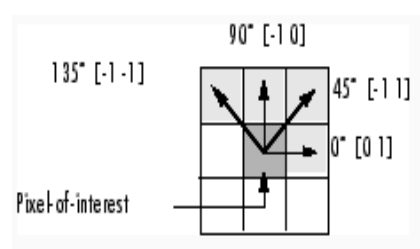

(b)

Figure 5. (a) GLCM Process Using Image I, (b) GLCM Direction and Offset. [1]

Table 1. Textural Feature Extracture by GLCM

\begin{tabular}{|c|c|c|}
\hline Feature name & Equation & Definition \\
\hline Contrast & Con $=\sum_{i=1}^{N_{g}} \sum_{j=1}^{N_{g}}(i-j)^{2} p(i, j)$ & Measure the local variance in GLCM \\
\hline Correlation & $\operatorname{Corr}=\frac{\sum_{i} \sum_{j}(i, j) p(i, j)-\mu_{x} \mu_{y}}{\sigma_{x} \sigma_{y}}$ & $\begin{array}{l}\text { Measure the joint probability occurance of the specified } \\
\text { pixel pairs. }\end{array}$ \\
\hline Energy & $\mathrm{E}=\sum_{i} \sum_{j} p(i, j)^{2}$ & Provides the sum of squared elements in the GLCM. \\
\hline Homogeneity & Homom $=\sum_{i} \sum_{j} \frac{1}{1+(i-j)^{2}} \mathrm{p}(\mathrm{i}, \mathrm{j})$ & $\begin{array}{l}\text { Measures the closeness of the distribution of elements in } \\
\text { the GLCM to the GLCM diagonal. }\end{array}$ \\
\hline
\end{tabular}

$(\mathrm{i}, \mathrm{j})$ represent the rows and columns, $\mathrm{p}(\mathrm{i}, \mathrm{j})$ denote the element from GLCM matrix, $N_{g}$ shows the number of different gray level in an image, $p_{x}(i)$ and $p_{y}(j)$ represent the marginal probability which is genereated by summation of rows and columns of GLCM. 
Table 2. Gradient Feature Extraction

\begin{tabular}{lcl}
\hline Feature name & \multicolumn{1}{c}{ Equation } & Definition \\
\hline Mean & $\mu=\sum_{i=1}^{n} p_{i} x_{i}$ & $\begin{array}{l}\text { Average of the values in the data set. } \\
\text { Average of the squared diffrences between the values } \\
\text { and the mean. }\end{array}$ \\
Standard deviation & $\sigma^{2}=\sum_{i=1}^{n} p_{i}\left(x_{i}-\mu\right)^{2}$ & Square root of the varience. \\
& $\boldsymbol{\sigma}=\sqrt{\sigma^{2}}$ &
\end{tabular}

\subsection{Classifier Construction}

After the feature generation it is necessary to develop a classifier using which true retinal area and artefact can be differentiate. So, RBFN have been applied. A radial basis function network is a special type of neural network which performs the classification using the input that is taken from the training set. A "prototype" is stored by each RBFN neuron. When a new data input has to classify then the Euclidean distance is computedbetween the prototype and the data input by each neuron. In simple words, if input is belongs to class A prototype than class B prototype then that input is classified as class A [12].

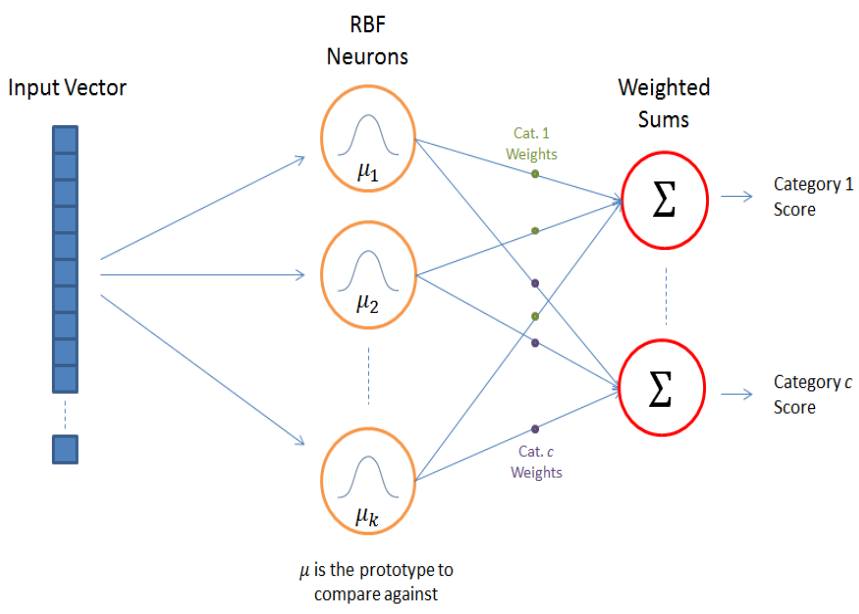

Figure 6. RBF Network Architecture [12]

The above Figure 6 shows the architecture of RBF network. This network consists of input vector, RBF neurons and the output vector.

Input vector: Classification of input vector is required which is $\mathrm{n}$-dimensional vector. The RBF neurons contains the entire input vector.

RBF Neurons: A prototype is stored in each RBF neurons. RBF neurons are compared with the input vector and its prototype. When the input is equal to its prototype then the output is taken as 1 . When the difference between. input and its prototype increases then the output response falls off 0 . So the output value lies between 0 and 1 which depends upon the similarity. The RBF neuron's shape is bell like structure which is shown in the above Figure 6.

Output nodes: A set of nodes is contained in the output network. Various scores are computed by each output node. The score is calculated using the weighted sum by the activation function from the RBF neuron. An output node is connected with weight value of the RBF neuron, before adding the activation value of the neuron is multiplied with the weight.

Each output node contain its own weight because the score for all category is computed by each output. The output node provide positive weight as well as negative weight. Positive weight has its one category and negative weight has another category.

$$
\mathrm{F}(\mathrm{x})=\frac{1}{\sigma \sqrt{2 \pi}} e^{-\frac{(x-\mu)^{2}}{2 \sigma^{2}}}
$$

Above equation is for one dimensional input where

$\mu=$ mean

$\boldsymbol{\sigma}=$ standard deviation 


$$
\mathrm{x}=\text { input }
$$

Slight different function is used in RBF activation function:

$$
\varphi=e^{-\beta(x-\mu)^{2}}
$$

$\mu$ refers to mean in the gaussian but here $\mu$ is the prototype vector which is belongs the bell curve center. Here $\varphi$ is used for the activation fuction. RBF Neuron Activation Function: The measurement of equality between the prototype and the input vector is calculated by RBF neuron [12].

\section{RESULTS}

We performed the experiment on an image which is obtained from Optos [4] and that are acquired by the ultrawide field SLO. Field of view (FOV) of each retinal image as shown in Figure 7 is upto $200^{\circ}$ with the resolution of $14 \mu \mathrm{m}$. The retinal image without dilation is captured by the device, over the pupil of $2 \mathrm{~mm}$ that is very small. There are two channels in the image i.e, red and green. The red channel whose wavelength is $633 \mathrm{~nm}$ displays the deeper structures of retina against choroid whereas the green channel whose wavelength

$532 \mathrm{~nm}$ gives information about the retinal pigment epithelium to the sensory retina. The dimension of each image is $3900 \times 3072$ and each pixel is showed by 8 bit on both green and red channels. The dataset is collected by diseased and healthy retinal images. Many diseased retinal images are collected from Diabetic Retinopathy patients [3]. The results and the accuracies of RBFN by dice-coefficient like evaluation metric. The amount of overlap between the benchmark acquired by the clinician and output of framework is called as Dice coefficient. Dice coefficient can be written as:

$$
\mathrm{D}(\mathrm{A}, \mathrm{B})=\frac{2|\mathrm{~A} \cap B|}{|A|+|B|}
$$

where, $A=$ image obtained by framework

$\mathrm{B}=$ image obtained by benchmark

$\mathrm{n}$ indicates the intersection and values varies in 0 and 1 where 1 is the higher value and 0 is the lower value.

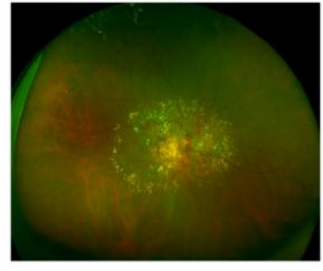

(a)

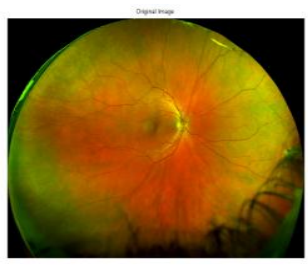

(d)

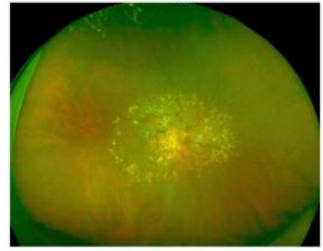

(b)

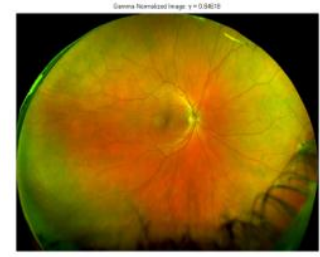

(e)

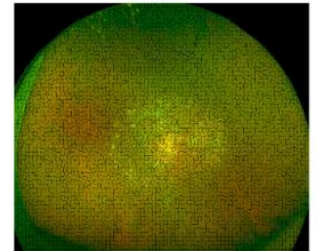

(c)

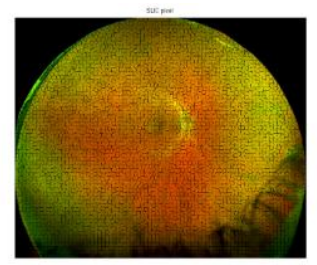

(f)

Figure 7. (a) and (d) Represent the Test (Original) Image from SLO Images (b) and (e) Represent the Gamma Normalized on the Original Images (c) and (f) Represent the Superpixel Classification Results 


\section{DISCUSSION AND CONCLUSION}

Extraction of retinal area from the SLO images are very imortant but difficult task. In this study, we proposed a technique using which true retinal area is detected from the SLO images. We have presented the preprocessing, superpixel generation, feature generation and classifier construction. SLICO is more faster, better performance and more memory efficient for generation of superpixel. Feature generation is very important step to reduce the computational cost. Then classifier construction is used to extract the retinal area. The RBFN classifier has been applied to achieve greater accuracy. Proposed work has been applied on 30 images and from all these images it has been concluded that the efficiency achieved is $96 \%$ but the computational time is little higher in case of RBFN with compared to ANN. This computational time can be reduced in the future.

\section{REFERENCES}

[1] M.J. Aligholizadeh, S. Javadi, R.S. Nadooshan, and K. Kangarloo, "Eyelid and eyelash segmentation based on wavelet transform for iris recognition”, in Proc. 4th Int. Congr. Image Signal Process, pp. 1231-1235, 2011.

[2] Thomas M. Deserno," Fundamentals of Biomedical ImageProcessing”, in Springer-Verlag Berlin Heidelberg of Biomedical Image Processing, 2011.

[3] Haleem, M.S., Han, L., van Hemert, J., Li, B. and Fleming, A., "Retinal Area Detector from Scanning Laser Ophthalmoscope (SLO) Images for Diagnosing Retinal Diseases", IEEE journal of biomedical and health informatics, 19(4), pp. 1472-1482, 2015.

[4] Optos. (2014). [Online]. Available: www.optos.com

[5] Seema Rani, Manoj Kumar, "Contrast Enhancement using Improved AdaptiveGamma Correction With Weighting Distribution Technique”, International Journal of Computer Applications, 101(11), pp. 0975 - 8887, 2014.

[6] Chollette C. Chude-Olisahl, Ghazali SUlong, Uche A.K. Chude-Okonkwo, Siti z. M. Hashim," Illumination Normalization for Edge-Based Face Recognition Using the Fusion of RGB Normalization and Gamma Correction", IEEE International Conference on Signal and Irnage Processing Applications, 2013

[7] A.K. Bhandaria, A. Kumara, G.K. Singh," Improved knee transfer function and gamma correction basedmethod for contrast and brightness enhancement of satellite image", International Journal of Electronics and Communications (AË̈), 69, pp. 579-589, 2015.

[8] http://ivrl.epfl.ch/research/superpixels\#SLICO

[9] Radhakrishna Achanta, Appu Shaji, KevinSmith, Aurelien Lucchi,Pascal Fua,and Sabine Su, "SLIC Superpixels Compared to State-of-the-Art Superpixel Methods", IEEE transactions on pattern analysis and machine intelligence, 34(11), 2274-2281, 2012

[10] M. Abra 'moff, W. Alward, E. Greenlee, L. Shuba, C. Kim, J. Fingert, and Y. Kwon, "Automated segmentation of the optic disc from stereo color photographs using physiologically plausible features”, Invest. Ophthalmol. Vis. Sci., vol. 48, pp. 1665-1673,2007

[11] http://in.mathworks.com/help/images/ref/graycomatrix.html\#responsive_offcanvas

[12] http://mccormickml.com/2013/08/15/radial-basis-function-network-rbfn-tutorial/

[13] M.S. Haleem, L. Han, J. van Hemert, and B. Li, "Automatic extraction of retinal features from colour retinal images for glaucoma diagnosis: A review”, Comput. Med. Imag. Graph. 37, pp. 581-596, 2013.

[14] R.C. Gonzalez and R. E. Woods, Eds., Digital Image Processing, 3rd ed. Englewood Cliffs, NJ, USA: PrenticeHall, 2006.

[15] D. Zhang, D. Monro, and S. Rakshit, "Eyelash removal method for human iris recognition", in Proc. IEEE Int. Conf. Image Process., pp. 285-288, 2006.

[16] A.V. Mire and B.L. Dhote, "Iris recognition system with accurate eyelash segmentation and improved FAR, FRR using textural and topological features", Int. J. Comput. Appl., 7, pp. 0975-8887, 2010.

[17] Y.H. Li, M. Savvides, and T. Chen, "Investigating useful and distinguishing features around the eyelash region", in Proc. 37th IEEE Workshop Appl. Imag. Pattern Recog., pp. 1-6, 2008

[18] B.J. Kang and K.R. Park, “A robust eyelash detection based on iris focus assessment”, Pattern Recog. Lett., 28, pp. 1630-1639, 2007.

[19] T.H. Min and R.H. Park, "Eyelid and eyelash detection method in the normalized iris image using the parabolic Hough model and Otsus thresholding method", Pattern Recog. Lett.30, pp. 1138-1143, 2009.

[20] H. Davis, S. Russell, E. Barriga, M. Abramoff, and P. Soliz, "Vision-based, real-time retinal image quality assessment", in Proc. 22nd IEEE Int. Symp. Comput.-Based Med. Syst., pp. 1-6, 20 